\title{
Response of Silicon photo-multipliers to a constant light flux
}

\author{
M. Caccia $^{\text {a* }}$, A. Bulgheroni ${ }^{\mathrm{a}}$, C. Cappellini ${ }^{\mathrm{a}}$, V. Chmill ${ }^{\mathrm{a}}$, M. Ramillia ${ }^{\mathrm{a}}$ F. Risigo ${ }^{\mathrm{a}}$ \\ ${ }^{a}$ Universitá dell'Insubria, Dipartimento di Fisica e Matematica \\ Via Valleggio 11, \\ 22100 Como (Italy)
}

The response of a Silicon Photomultiplier to a constant illumination has been interpreted in term of GeigerMueller avalanche frequency, actually correlated to the photon flux via the photon detection efficiency. The hypothesis has been verified in laboratory tests and applied throughout the development of a device for real-time dosimetry in mammography.

\section{INTRODUCTION}

Silicon Photo-Multipliers (SiPM hence-over) do represent the state of the art for low visible light flux measurements with single photon sensitivity $[1,2]$. Whether most of the envisaged applications are related to pulsed light $[3-5]$, the response to a constant light field is certainly of interest. This was investigated within RAPSODI (Radiation Protection with Silicon Optoelectronics Devices and Instruments), a collaborative research project ${ }^{2}$ supported by the European Commission within the $6^{\text {th }}$ Framework Program. The study was linked to one of the applications addressed in the project, namely the development of a real-time dosimeter for mammography. The methodology, the laboratory tests and the application to the mammography dosimeter prototype characterization are reported in the following.

\section{DETECTOR CHARACTERIZATION}

Whenever SiPM are being integrated into instruments probing a constant light flux, the key figures of merit are the linear dynamic range and the minimum detectable signal, the latter being essentially limited by the Dark Count Rate (DCR). As long as the light intensity increases and the mean time of arrival between photons is comparable to the single avalanche development

\footnotetext{
*Corresponding author; Massimo.Caccia@uninsubria.it

${ }^{2}$ contract number Coop-32993
}

time, the detected signal corresponds to piled-up events, eventually deviating from the linearity as the probability to hit a cell during the recovery time is non-negligible. In a pile-up situation, the information on the light intensity is actually measured by the current at the output node, interpreted in the following in terms of Geiger-Mueller (GM) avalanche frequency. Whether proven to be true, this hypothesis allows to link the macroscopic system response to occurrencies at the detector single cell level in a quantitive way, providing a useful tool for the choice of the optimal detector characteristics.

The features of the sensor of interest, produced by $\mathrm{SENsL}^{3}$, are reported in Table 1 . The I-V curve directly measured using a Source and Measurement Unit integrated into a Keithley 4200 Semiconductor Analyzer System is shown in Fig.1. The brench of the curve beyond the breakdown was presumed to be dominated by the current originating from the avalanches triggered by the thermally generated carriers in the junction region, namely the Dark Count Rate (DCR). The hypothesis was verified measuring independently the DCR, the cross talk and the sensor gain and calculating the current as:

$I=G_{S i P M} e^{-} \nu_{A}\left(1+X_{\text {talk }}\right)$,

\footnotetext{
${ }^{3} \mathrm{SENsL}$ is a RAPSODI partner company; address: Lee House, Riverview Business Park, Bessboro Road, Blackrock, Cork
} 


\begin{tabular}{lc}
\hline \hline Sensor ID: & CSI 0747015 A20 HD \\
\hline Number of Diodes: & 8640 \\
Single cell dimension: & $20 \mu \mathrm{m} \times 20 \mu \mathrm{m}$ \\
Fill Factor: & $43 \%$ \\
Breakdown Voltage: & $28.04 \mathrm{~V}$ \\
Leakage current: & $0.95 \mu \mathrm{A}$ \\
Dark Count Rate: & $(8 \pm 0.9) \mathrm{MHz}$ \\
Optical Crosstalk: & $(22.5 \pm 3.5) \%$ \\
Gain: & $(1 \pm 0.04) \times 10^{6}$ \\
PDE: & $(3.3 \pm 0.3) \%$ \\
\hline \hline
\end{tabular}

Table 1

Parameters of the SensL 9k SiPM. Values were measured at room temperature and $+2 \mathrm{~V}$ biasing over the breakdown voltage; the Photon detection efficiency (PDE) has been estimated taking into account crosstalk effects, and it refers to an illumination with $510 \mathrm{~nm}$ green light.

where $e^{-}$is the electron charge, $G_{S i P M}$ is the gain of the SiPM, $X_{\text {talk }}$ is the cross-talk, and $\nu_{A}$ is the GM avalanche frequency. The cross-talk was defined as the ratio of the DCR at "half photoelectron" threshold and "one-and-half photoelectrons" threshold and $\nu_{A}$, in this peculiar situation of no light exposure, corresponds to $D C R_{0.5}$, the dark count rate at "half photoelectron" threshold. DCR values are summarized in Table 2. In view of the specific application reported in the following section, the current was also measured indirectly by normalizing the charge integrated by a CAEN V792 Charge to Digital converter (QDC) over a well defined gate window. Values of the current for every specific biasing voltages were obtained as:

$I=Q D C_{\text {cal }} \frac{\Delta Q D C}{t_{\text {gate }}} \frac{1}{K_{a m p}}$,

where $Q D C_{c a l}=0.11 p C /$ channel is the QDC calibration constant; $\triangle Q D C$ is the most probable value of the integrated charge spectrum; $t_{\text {gate }}$ is the integration time and $K_{a m p}=470 / 100$ is the effective amplification factor of the transimpedence amplifier.

Data from the direct and indirect measurements are overlaid in Figure 2, showing a remarkable

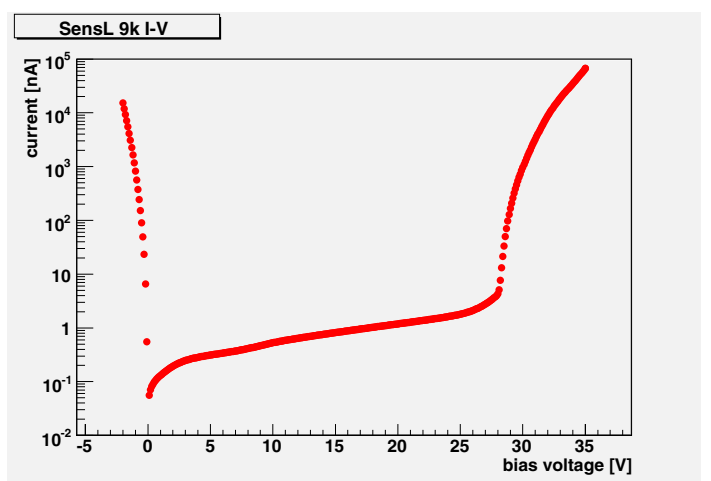

Figure 1. I-V profile of the SensL CSI 0747015 SiPM (SensL9k henceover); mesurements were performed at $25.6{ }^{\circ} \mathrm{C}$.

agreement and confirming the possibility to interpret the current at the output node in terms of GM avalanche frequency.

\section{REAL-TIME DOSIMETRY IN MAM- MOGRAPHY: BASIC DETECTOR IS- SUES}

Mammography identifies the radiographic examination of the human breast, targeted to the early diagnostics of different types of tumours, cysts and micro-calcifications. Mammography is the only imaging technique that has been proven to reduce mortality from breast cancer. Hence, together with self-breast and physician examination, it is an essential part of regular breast care. In some countries, routine mammography of women is encouraged every 1 to 5 years. It is clear that knowing the actual dose released to the patient during any irradiation is of extreme importance, especially in the case of screening procedures. Within RAPSODI, a novel real time dosimeter for mammography has been developed. The new device (identified as MAMMODOS in the following, protected by an international patent application ${ }^{4}$ ) is a novelty in the field of mammography. It is a cost-effective

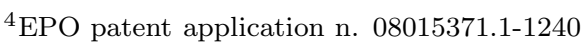




\begin{tabular}{ccccc}
\hline \hline Bias $[\mathrm{V}]$ & DCR $_{0.5}[\mathrm{MHz}]$ & DCR $_{1.5}[\mathrm{MHz}]$ & $X_{\text {talk }}(\%)$ & Current $[\mu \mathrm{A}]$ \\
\hline 29.5 & $7.1 \pm 0.8$ & $0.9 \pm 0.1$ & $12.7 \pm 2$ & $0.82 \pm 0.10$ \\
30.0 & $8.0 \pm 0.9$ & $1.8 \pm 0.2$ & $22.5 \pm 3.5$ & $1.53 \pm 0.18$ \\
30.5 & $10.2 \pm 1.1$ & $3.2 \pm 0.4$ & $31.4 \pm 4.8$ & $2.80 \pm 0.34$ \\
31.0 & $12.0 \pm 1.3$ & $5.3 \pm 0.6$ & $44.2 \pm 6.8$ & $4.53 \pm 0.56$ \\
31.5 & $14.0 \pm 1.5$ & $8.0 \pm 0.9$ & $57.1 \pm 8.8$ & $6.94 \pm 0.87$ \\
\hline \hline
\end{tabular}

Table 2

DCR and $X_{\text {talk }}$ values for the SensL 9k. Measurements performed at room temperature.

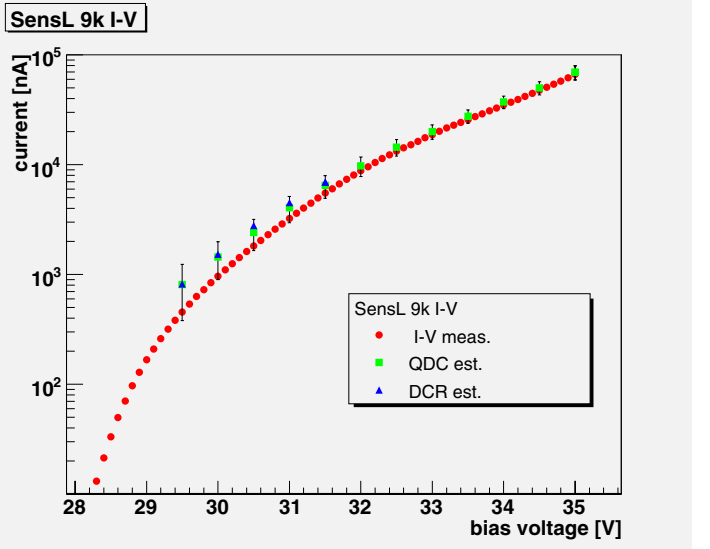

Figure 2. I-V data for the Sensl 9k sensor; indirect measurement from the QDC data are shown together with an estimate from the Dark Count Rate. The reference points refer to the direct measurement.

and compact instrument, consisting in a very thin scintillating sensitive volume, a SiPM sensor measuring the scintillating light and a small local display. The part introduced into the imaging beam is being engineered to be fully tissue equivalent and homogeneous, to avoid introducing any artifacts in the image. The instrument is designed to be used in contact with the patient due to the absence of any electrical elements in the sensor part, mounted on the compression paddle of the mammography X-ray machine.

Several prototypes were constructed and qualified; results reported here refer to two different geometries, with a sensitive blue emitting scintillating volume consisting in either a thin tile or in a few centimeter long fiber. Scintillators were optically coupled to a $1 \mathrm{~mm}$ diameter Wave Length Shifting (WLS) fiber from blue to green, conveying the light to a SiPM matching the sensor peak spectral sensitivity. The current produced in the SiPM by the X-ray burst was amplified by a transimpedence circuit developed at SENsL characterised by an effective gain of $470 \mathrm{mV} / \mathrm{mA}$ and digitized by a CAEN V792 Charge to Digital Converter (QDC) during a well defined integration time. The signal was typically sampled at a frequency of $30 \mathrm{kHz}$ with integration times ranging between 500 and $5000 \mathrm{~ns}$.

Measurements have been performed at the calibration laboratories located at PTW-Freiburg ${ }^{5}$, a RAPSODI partner company. Irradiations have been performed using the Siemens-PANDOROS X-ray machine, characterized by the possibility to set different dose rates by changing the anode current values. Dose rates spanning from a few up to hundreds of $\mathrm{mGy} / \mathrm{s}$ have been used to irradiate the prototypes according to the enduser requirements. For each irradiation, the effective dose rate and the irradiation time were simultaneously measured with calibrated detectors produced by PTW. The typical output signal from the system is displayed in Figure 3, where the samples corresponding to the tile irradiation are clearly distinguishable. Off-beam events have been used to estimate the pedestal value and its spread, for further use in the analysis chain. Xray irradiation in mammography lasts longer than $1 \mathrm{~ms}$, a time lapse long enough to analyze the re-

${ }^{5}$ PTW-Freiburg, Lorracher Strasse 7, 79115 Freiburg, Germany 


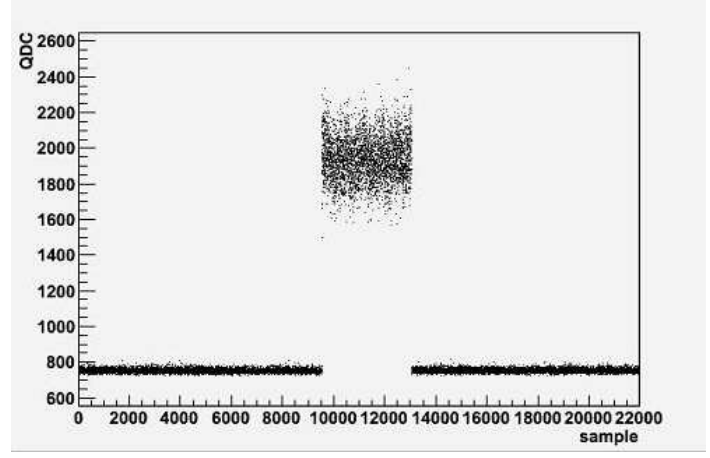

Figure 3. The signal for a typical data set is shown as a function of the sample number. The integration time for the reported data was 3000 ns for a dose rate of $21 \mathrm{mGy} / \mathrm{s}$.

sponse of SiPM in the hypothesis of a constant flux. The sensitive volume and the SiPM characteristics have been matched in order to guarantee the end-user specifications in terms of repeatability, precision, sensitivity, minimum detectable signal and linearity against the dose rate in the region of interest $(1-200 \mathrm{mGy} / \mathrm{s}$ as of the protocol IEC 61674). Preliminary results have been shortly reported in [6] and the full comprehensive analysis will be the subject of a forthcoming publication.

As exemplary illustration relevant for this paper, an analysis of the GM avalanche frequency induced by the X-ray burst was performed following the method illustrated in the previous section and entered the design optimization. The minimum value of the current $(0.786 \pm 0.005 \mu \mathrm{A})$ was recorded for the prototype based on a scintillating fiber tip and a dose rate provided by the machine of $5.93 \pm 0.02 \mathrm{mGy} / \mathrm{s}$. The value was calculated according to Eq. 2, for an integration time of $5000 \mathrm{~ns}$ The maximum measured current was $147.40 \pm 0.09 \mu \mathrm{A}$, irradiating the scintillating tile with a dose rate of $194.9 \pm 1.5 \mathrm{mGy} / \mathrm{s}$, the maximum value available with the machine being used. As of Eq. 1, the corresponding GM avalanche frequencies were measured to be $4.1 \pm$
$0.2 \mathrm{MHz}$ for the lowest value and $770 \pm 35 \mathrm{MHz}$ for the highest value, being the errors calculated taking into account as well the uncertainties on $G_{S i P M}$ and $X_{\text {talk }}$.

These results ruled out the SENsL sensor in use for the fiber tip option, where the combination of the low PDE and high DCR did not guarantee a minimum detectable signal at the $1 \mathrm{mGy} / \mathrm{s}$ level; on the other hand, the detector was optimal for the scintillating tile option, essentially in view of the large number of cells reducing to the $10^{-5}$ level the probability to have a photon hitting a cell during its recovery time, presumed to be at the $50 \mathrm{~ns}$ level. As a matter of fact, the set-up based on the tile and the sensor under test fulfilled the criteria, with a measured precision of $3.04 \pm 0.05 \%$, a minimum detectable signal of $0.524 \pm 0.009 \mathrm{mGy} / \mathrm{s}$, a sensitivity of $2.60 \pm 0.05$ $\mathrm{mGy} / \mathrm{s}$ and a deviation from the linearity over the full range not exceeding $10 \%$. These results completed the feasibility study and started the engineering phase, close to completion by the time of writing.

\section{CONCLUSIONS}

The use of Silicon photomultipliers to probe constant illumination fields requires a characterization in different terms with respect to the response to pulsed light, requiring the development of a complementary model and method. A link between the macroscopic response in terms of electrical current and the elementary occurrencies at single cell level was experimentally established interpreting the current in terms of GeigerMueller avalanche frequency. The method was proven to be helpful in evaluating the properties of the sensor to be integrated in a real-time dosimeter for mammography. The sensor being considered, featuring about 9000 cells, was proven to be linear for a Geiger-Mueller avalanche frequency exceeding $800 \mathrm{MHz}$.

\section{REFERENCES}

1. A. Adikinov et al., Nucl. Inst. And Meth. A 387 (1997) 231-234 
2. P. Buzhan et al., Nucl. Inst. And Meth. A 504 (2003) 48-52

3. V. Andreev et al.,Nucl. Inst. And Meth. A 540 (2005) 368-380

4. D.J. Herbert et al., Nucl. Inst. And Meth. A 573 (2007) 84-87

5. G.LAurenti et al., et al., Nucl. Inst. And Meth. A 588 (2008) 267-271

6. F. Risigo et al., Proceedings of the IWORID 2008 conference, to bepublished on Nucl. Inst. And Meth. A 